\title{
Síndrome de Mirizzi tipo V-um desafio diagnóstico e de prognóstico alarmante na colelitíase: relato de caso
}

\section{Mirizzi type $V$ syndrome - a diagnostic test and alarming prognosis in cholelithiasis: case report}

Ana Paula Bastos ${ }^{\dagger *}$, Lucineide Martins de Oliveira Maia

Como citar esse artigo. Bastos, A.P Maia, L.M.O. Síndrome de Mirizzi tipo V - um desafio diagnóstico e de prognóstico alarmante na colelitíase: relato de caso. Revista de Saúde. 2019 Jul./Dez; 10 (2): 56-60.

\begin{abstract}
Resumo
Decorrente de uma complicação tardia da colelitíase, a Síndrome de Mirizzi é definida como uma obstrução extrínseca do ducto hepático comum devido a cálculos impactados no ducto cístico ou no infundíbulo da vesícula, que acarreta em uma sintomatologia semelhante à colelitíase. O presente artigo objetiva relatar um caso raro de paciente portadora de Síndrome de Mirizzi tipo V, diagnosticada no intra-operório de colecistectomia. Realizou-se uma revisão nas bases de dados Scielo, Lilacs e Pubmed na busca de artigos relacionados. Esta patologia, de difícil diagnóstico pré-operatório, possui uma classificação, a Csendes, que subdivide a doença em cinco grandes grupos e determina, a partir desta, a melhor técnica cirúrgica a ser empregada. O caso clínico relatado apresenta uma lesão clinicamente indistinguível da colelitíase, que necessitou de um tratamento cirúrgico na tentativa de uma terapia curativa.
\end{abstract}

Palavras-chave: Síndrome de Mirizzi, colelitíase, colecistectomia, Csendes.

\begin{abstract}
Due to a late complication of cholelithiasis, Mirizzi Syndrome is defined as an extrinsic obstruction of the common hepatic duct due to gallstones impacted on the cystic duct or gallbladder infundibulum, leading to a cholelithiasis-like symptom. This article aims to report a rare case of a patient with Mirizzi syndrome type V, diagnosed intraoperatively with cholecystectomy. A review of Scielo, Lilacs and Pubmed databases was performed for related articles. This pathology, which is difficult to diagnose preoperatively, has a classification, Csendes, which subdivides the disease into five major groups and determines, from this, the best surgical technique to be employed. The reported case report presents a clinically indistinguishable lesion of cholelithiasis, which required surgical treatment in the attempt of curative therapy.

Keywords: Mirizzi Syndrome, cholelithiasis, cholecystectomy, Csendes.
\end{abstract}

\section{Introdução}

Descrita pela primeira vez por Pablo Mirizzi, em $1948^{1}$, a síndrome que leva o seu nome, é decorrente de uma complicação tardia da colelitíase, em que um cálculo impacta no ducto cístico ou na bolsa de Hartman, obstruindo mecanicamente o ducto biliar comum $^{2}$. A partir desse momento, vários fenômenos são desencadeados, sendo os mais comuns: dor abdominal, icterícia, colangite ${ }^{3}$ e fístula colecistobiliar ${ }^{1}$. Considerada uma afecção incomum, acometendo de 0,3 a $5,7 \%$ da população ${ }^{2}$, é mais prevalente em mulheres de idade avançada. De acordo com a classificação de Csendes, é dividida em cinco grandes grupos. A do tipo I corresponde à que ocasiona compressão extrínseca do ducto hepático comum ou do colédoco por cálculo presente no colo vesicular ou ducto cístico ${ }^{3}$. Já a do tipo II é descrita como que contém uma fístula colecistobiliar com erosão de diâmetro menor que $1 / 3$ da circunferência do ducto hepático comum ou do colédoco ${ }^{3}$. Caso esta mesma fístula ultrapasse $2 / 3$ do diâmetro do ducto hepático comum ou do colédoco ${ }^{3}$, é classificada como sendo do tipo III. A classificação IV ocorre quando a fístula colecistobiliar atinge toda a circunferência do ducto hepático comum ou do colédoco. Por fim, inserida recentemente na classificação, a do tipo $\mathrm{V}$ é descrita quando se tem uma associação entre qualquer uma das relatadas anteriormente mais a presença de uma fístula colecistoenérica ${ }^{3}$. Esta última sofre ainda uma subdivisão: a Va, na qual não se observa o íleo biliar e a $\mathrm{Vb}$ em que há a presença do íleo-biliar³. A 
Síndrome de Mirizzi é dificilmente diagnosticada no pré-operatório, já que sua apresentação clínicolaboratorial não é específica. A sua não detecção precoce é responsável por altos índices de lesões no ducto biliar durante o procedimento cirúrgico ${ }^{3}$. O presente artigo relata um caso raro de paciente portadora de Síndrome de Mirizzi tipo V, diagnosticada no intra-operório de colecistectomia

\section{Relato De Caso}

J.D.M, sexo feminino, 68 anos, aposentada e natural de Mendes - RJ, deu entrada no Hospital Universitário de Vassouras (HUV) com queixa de dor abdominal, de forte intensidade, do tipo cólica, localizada em hipocôndrio direito, que se iniciou há 10 meses. Na anamnese, referiu piora da dor, acompanhada de náuseas e vômitos, após ingesta de alimentos gordurosos. Negou icterícia prévia, colúria e acolia fecal.

Mediante estes achados, foi solicitada uma ultrassonografia de abdome total, por meio da qual evidenciou-se vesícula biliar contraída, de paredes finas e lisas, repleta de cálculos com projeção de sombra acústica posterior. A paciente foi então, encaminhada para a realização do risco cirúrgico e, posteriormente, teve marcada a colecistectomia.

Nac olecistectomia, realizada com incisão de Kocher, foi visualizado um processo inflamatório local e aderências que se estendiam do infundíbulo vesicular ao ducto do colédodo. Além disso, havia a presença de

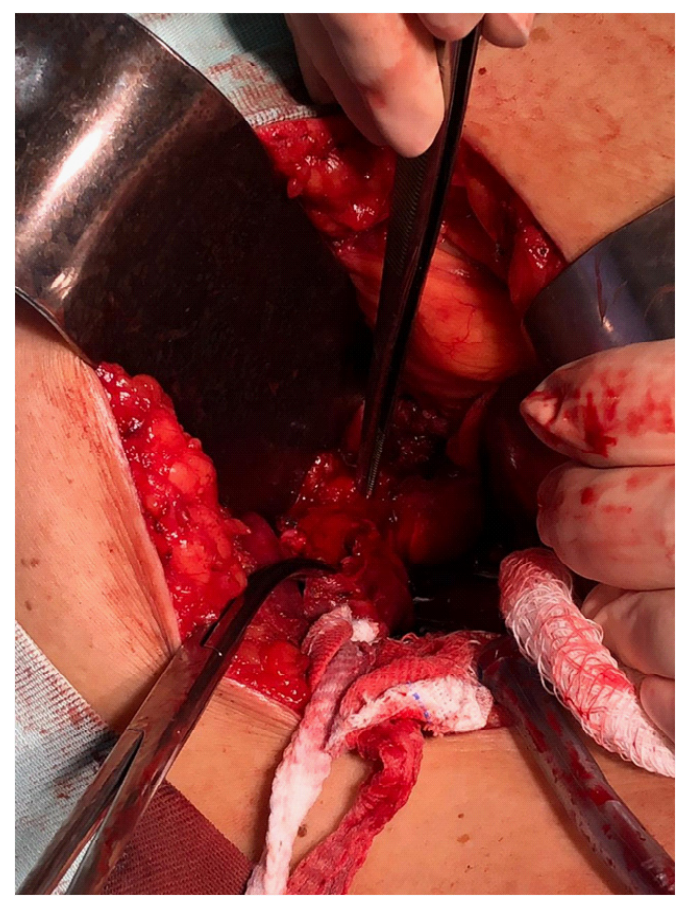

Figura 1. Presença da fístula colecistoduodenal diagnosticada intra-operatório durante a colecistectomia. cálculos na vesícula biliar e fístulas colecistobiliar e colecistoduodenal (figura 1). O quadro encontrado foi compatível com uma Síndrome de Mirizzi tipo V.

A partir dos achados, realizou-se lise das aderências com liberação da fístula duodenal e dissecção da parede vesicular. Foi ainda identificado um ducto cístico, com posterior realização de colangiografiaintraopertória (figura 2), que evidenciou uma dilatação das vias biliares intra e extra-hepática. Foi realizada drenagem da via biliar principal com colocação de dreno de Kehr. Ao término da cirurgia, a paciente foi internada na Unidade de Terapia Intensiva (UTI) do HUV.

Seis dias após a colecistectomia, a paciente evoluiu com piora clínica, desidratação, leucocitose e saída de secreção purulenta em grande quantidade da ferida operatória, justificando a indicação de nova laparotomia, que evidenciou a presença de abscesso intracavitário e coleperitôneo por deiscência parcial da sutura duodenal. Realizou-se então nova drenagem das vias biliares, além de uma nova rafia duodenal. Após a cirurgia, iniciou-se terapia com ceftriaxona e metronidazol, devido a alterações clínicas sistêmicas e laboratoriais (presença de leucocitose e desvio para a esquerda). A paciente evoluiu novamente com piora clínica e saída de secreção de aspecto bilioso pela lateral da ferida operatória, sendo o esquema de antibiótico anterior, substituído por meropenem, um antibiótico mais forte, já que não apresentou melhora clínica do quadro.

No décimo primeiro dia de internação na UTI, em grave estado geral, a mulher evoluiu com icterícia $(3+/ 4+)$ e dor abdominal. Mediante ao quadro, outra laparotomia foi realizada, com nova colangiografia e visualização de grande cálculo de colédoco (figuras 3 e 4). Seguiu-se com exploração das vias biliares, coledocotomia e retirada do cálculo com $4 \mathrm{~cm}$ em seu maior diâmetro (figuras 5 e 6). Após reabordagem, cursou com instabilidade hemodinâmica, necessitando de infusão de noradrenalina e ventilação mecânica. $\mathrm{O}$ esquema com meropenem foi mantido. A icterícia persistiu mesmo após a retirada do novo cálculo das vias biliares.

A partir do décimo quarto dia de internação, observou-se piora da função renal o que justificou prescrição de furosemida a fim de melhorar seu débito urinário, bem como saída de secreção sugestiva de bile pela ferida operatória, configurando novo coleperitôneo. A dose do meropenem foi reajustada de acordo com a função renal e iniciado uso do antibiótico vancomicina, devido à piora do leucograma, que apresentou um desvio para esquerda, caracterizando um quadro de sepse abdominal. Dois meses após, a paciente evoluiu com uma parada cardiorrespiratória, vindo a óbito. 


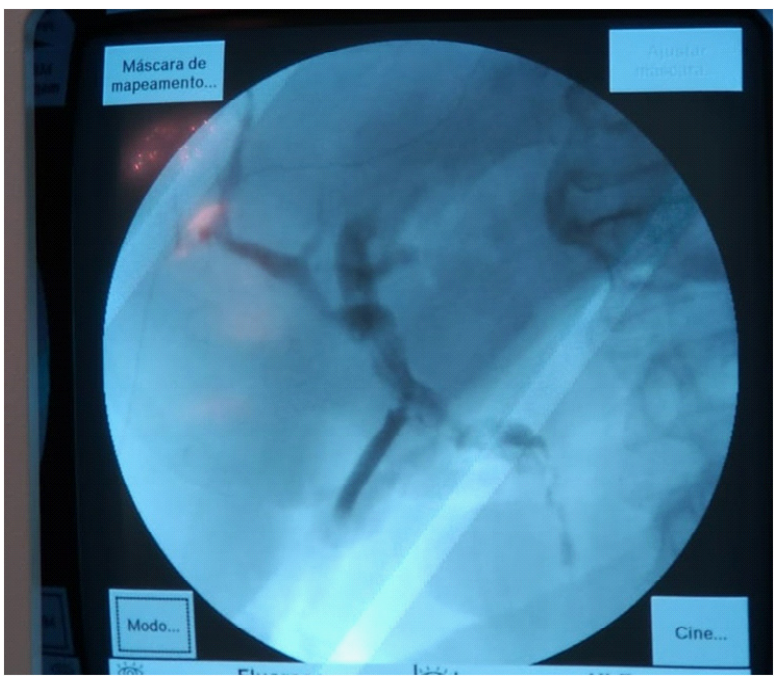

Figura 2. Colangiografia intra-opertória com dilatação das vias biliares.

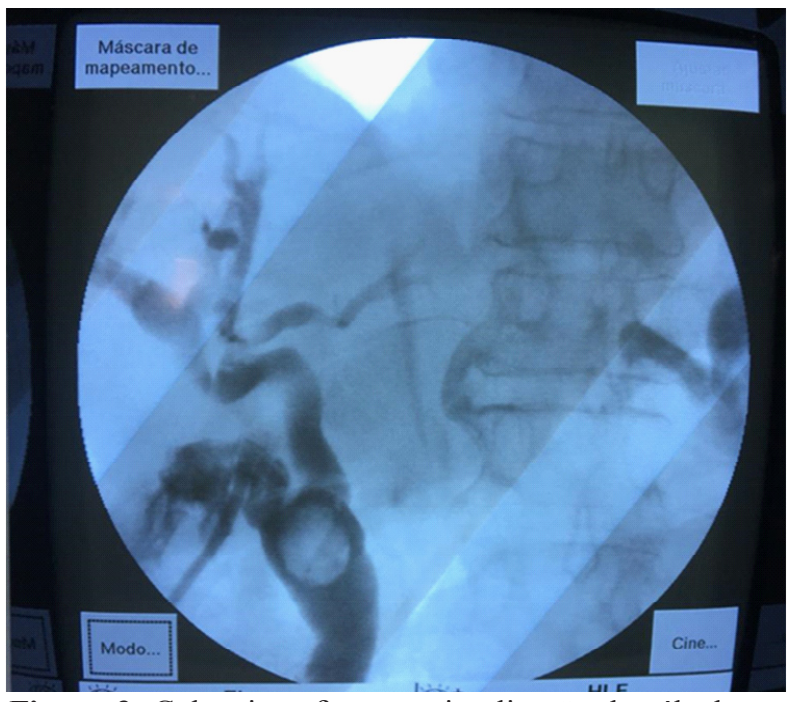

Figura 3. Colangiografia com visualização de cálculo em colédoco.

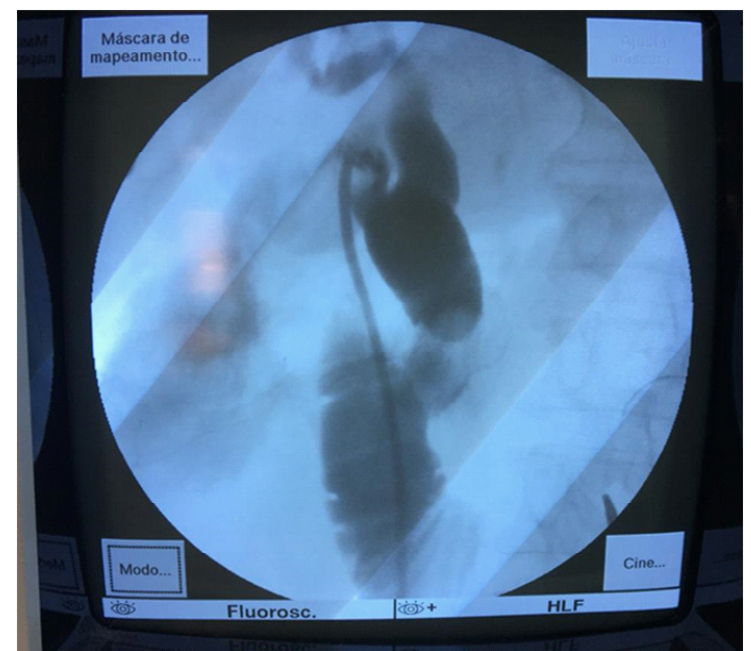

Figura 4. Colangiografia com visualização de cálculo em colédoco.

\section{Materiais e Métodos}

Após assinatura do Termo de Consentimento Livre e Esclarecido (TCLE) pela paciente e aprovação da solicitação de campo de pesquisa pela Direção do Centro de Estudos do Hospital Universitário de Vassouras foi realizada uma coleta de dados a partir do prontuário médico. $\mathrm{O}$ referencial teórico utilizou informações disponíveis nas bases de dados Lilacs, Scielo e Pubmed, com os descritores Síndrome de Mirizzi, colelitíse, Mirizzi Syndrome, cholelithiasis, colecistectomia e classificação de Csendes, entre o período de 2008 a 2019.

\section{Discussão}

Complicação rara e tardia da doença do cálculo biliar, a Síndrome de Mirizzi é definida como sendo uma obstrução extrínseca do ducto hepático comum devido a cálculos impactados no ducto cístico ou no infundíbulo da vesícula ${ }^{4}$. Os sintomas são semelhantes à colelitíase aguda, podendo a patologia vir ou não, acompanhada de diarreia e icterícia.

Os diagnósticos, tanto da fístula quanto da Síndrome de Mirizzi, devem ser feitos preferencialmente no pré-operatório, devido aos altos índices de morbidade e mortalidade relacionada a doença ${ }^{2}$. Porém, torna-se difícil, devido seus achados não específicos. No caso em questão, a paciente apresentou a sintomatologia clássica da colelitíase e o diagnóstico da fístula colecistoduodenal foi realizado no período intra-operatório, o que aumentou os riscos de mortalidade.

Apesar das mais diversas e modernas técnicas de imagens disponíveis, o diagnóstico desta Síndrome por meio destas ainda torna-se limitado. $\mathrm{O}$ raio $\mathrm{X}$ simples de abdome, por exemplo, não permite a detecção da enfermidade, porém, auxilia nos diagnósticos diferenciais por permitir a visualização de muitos sinais indiretos. Os que possuem maior especificidade e sensibilidade ${ }^{5}$ para a suspeita ou diagnóstico da 


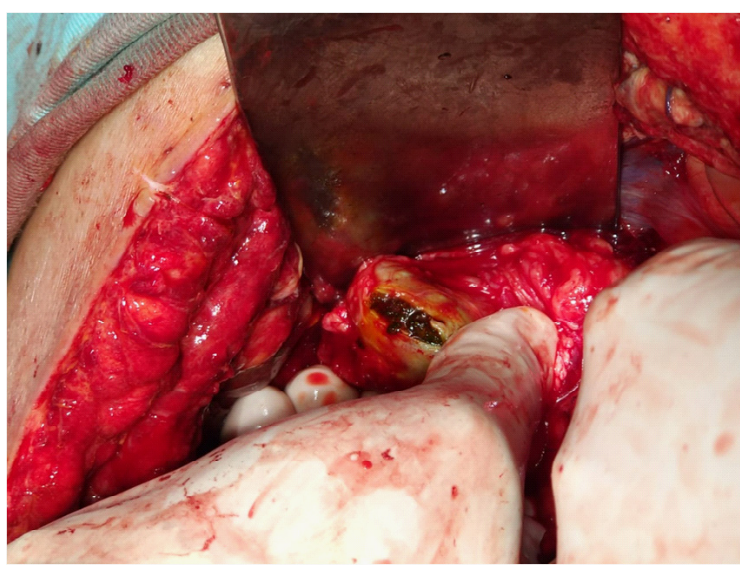

Figura 5. Terceira laparotomia com exploração e retirada de cálculo da via biliar.

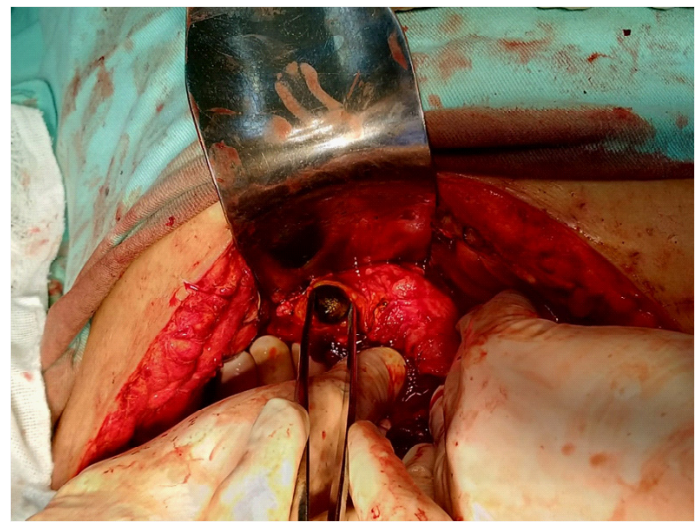

Figura 6. Cálculo sendo retirado da via biliar após exploração através da laparotomia.

Síndrome de Mirizzi são a tomografia helicoidal, a colangiorressonância e a colangiopancreatografia retrógrada endoscópica. Porém, esses exames de maior acurácia, na maioria dos casos não se justificam, já que a sintomatologia da doença e da fístula são semelhantes às da colelitíase, não tendo algo específico que sugira uma maior investigação. Todos os exames complementares citados não foram realizados na paciente, pois a sintomatologia a qual apresentou, era compatível com uma colelitíase, e o diagnóstico desta é clínico, ou seja, não necessita de nenhum exame complementar.

O tratamento da Síndrome de Mirizzi é cirúrgico e deve ser guiado por princípios como: dissecção cuidadosa da via biliar, retirada completa dos cálculos presentes no trato biliar, identificação do ducto hepático comum e pela colangiografia intra-operatória ${ }^{6}$. O tipo de cirurgia empregada é altamente dependente do grau de deformidade anatômica encontrada, assim como o grau de inflamação ${ }^{6}$. Contudo, há técnicas de escolha de acordo com cada estágio da classificação de Csendes.

No tipo I de Csendes, que corresponde na compressão extrínseca do ducto hepático comum ou do colédoco por cálculo presente no colo vesicular ou ducto cístico $^{3}$, o tratamento de escolha é a colecistectomia ${ }^{6}$ com a remoção dos cálculos. Já as alterações classificadas como II e III, onde existe a presença de fístula colecistobiliar, atingindo menos que $1 / 3$ e mais que $2 / 3^{3}$, respectivamente da circunferência do ducto hepático comum ou do colédoco, a opção cirúrgica é pela técnica de Sandblon ${ }^{6}$, com abertura do fundo da vesícula para a retirada dos cálculos. Além da técnica, está empregada ainda a colocação do dreno de $\mathrm{Kerh}^{6}$ no ducto hepático comum, logo acima da área que foi reparada. Devido ao alto grau de comprometimento da anatomia da árvore biliar, na Síndrome de Mirizzi tipo IV, está indicado uma hepaticojejunostomia ou coledocoduodenostomia ${ }^{6}$.

Quando se diagnostica a fístula colecistoentérica, a tipo $\mathrm{V}$ de Csendes, os principais órgãos envolvidos são o estômago, duodeno e cólon ${ }^{3}$. Para o caso descrito, a paciente em questão apresentava um envolvimento do duodeno, segundo órgão mais acometido nesta classificação. O manejo, caso haja a presença de íleo biliar é a enterolitotomia e fechamento da fístula. Porém, se este não estiver presente, faz-se apenas o fechamento do orifício da fístula ${ }^{3}$, sendo esta a técnica empregada na paciente relatada. A opção cirúrgica laraposcópica também é empregada para todas as classificações da Síndrome de Mirizzi, exceto para o estágio V, em que não há dados suficientes relatados ${ }^{2}$.

\section{Considerações Finais}

Apesar de a sintomatologia ser semelhante ao da colelitíase, os diagnósticos da Síndrome de Mirizzi e da fístula devem ser, preferencialmente, feitos ainda no pré-operatório, para que as taxas de mortalidade não se elevem. O caso apresentado trata-se de uma enfermidade rara, que necessita de avanços médicos e tecnológicos para o seu diagnóstico precoce.

A cirurgia, o único tratamento imposto à doença, é complicado e por vezes, controverso, na qual exige um cuidado maior devido à presença das alterações anatômicas feitas pela Síndrome.

\section{Referências}

1. Beorlegui JSB, Laguna EM, Gil-Albarellos PB, Sánchez RC, de Marcos NM, Sancho AA. Síndrome de Mirizziasociado a lacolelitiasis complicada delanciano: diagnóstico y tratamento laparoscópico. RevGastroent Per, 2008; 28: 15-21.

2. Yetisir F, Sarer AE, Acar HZ, Parlak O, Basaram B, Yazicioglu O. Laparoscopic resection of cholecystocolic fistula and subtotal cholecystectomy by tri-staple in a type V Mirizzi Syndrome. Case Report in Hepatol, 2016; 1-4.

3. Lacerda PS, Ruiz MR, Melo A, Guimarães LS, Junior RAS, Nakajima GS. Síndrome de Mirizzi: um grande desafio cirúrgico. ArqBrasCirDig, 2014; 27(2): 221-231.

4. Neto OCLDF, Pedrosa MGL, Miranda AL. Manejo cirúrgico da Síndrome de Mirizzi. ArqBrasCirDig, 2008; 21 (2): 51-4.

5. Gil JMG. El síndrome de Mirizzi, um desafio para elciirurjano. 
RevCubCir, 2016; 55(2): 151-163.

6. Ferreira RM. Síndrome de Mirizzi: relato de caso. NetSaber artigos [homepage na internet]. Acesso em 2 jan 2019. Disponível em: http://artigos. netsaber.com.br/resumo_artigo_36776/artigo_sobre_sindrome-de-mirizzi-relato-de-caso 